\title{
Spectral target classification with SOSTAR-X
}

\author{
M.P.G. Otten ${ }^{\# 1}$, E. de Koster ${ }^{* 2}$, \\ J.S. Groot ${ }^{\# 3}$, A.J. de Jong ${ }^{\# 4}$, M. van den Akker ${ }^{* 5}$, P. Vriend ${ }^{* 6}$ \\ ${ }^{\#}$ TNO Defence, Security and Safety \\ P.O. Box 96864, 2509 JG The Hague, The Netherlands \\ matern.otten@tno.nl \\ ${ }^{3}$ jos.groot@tno.nl \\ ${ }^{4}$ ardjan.dejong@tno.nl \\ ${ }^{*}$ Dutch Space \\ P.O. Box 32070, 2303 DB Leiden, The Netherlands \\ 2e.de.koster@dutchspace.nl \\ ${ }^{5} \mathrm{~m} . v d . a k k e r @ d u t c h s p a c e . n l$ \\ ${ }^{6} \mathrm{p}$.vriend@dutchspace.nl
}

\begin{abstract}
The SOSTAR-X project has developed a demonstrator dedicated to the surveillance of both fixed and moving targets on large coverage areas, target activity analysis on dedicated areas and classification on designated targets. In this project, The Netherlands contribution has been in the areas of Spectral Classification (SPC mode), SAR SPOT mode autofocus, antenna harness, and ground target coordination during the flight campaign in the Netherlands. This paper focuses on the topic of Spectral Classification. The so-called SPC mode is a dedicated mode activated on a designated target, which classifies the target as wheeled, tracked (treaded), rotary wing, or rotating antenna. This is achieved by producing a high resolution Doppler profile, containing modulations caused by moving parts of the vehicle, and classifying on the basis of this profile. The SPC mode has been designed, implemented in the sensor and the Radar Processing Unit, and validated during extensive flight trials, in the Netherlands, Germany, and France.
\end{abstract}

\section{INTRODUCTION}

The SOSTAR-X project has developed a demonstrator dedicated to the surveillance of both fixed and moving targets on large coverage areas, target activity analysis on dedicated areas and classification on designated targets.

In this project, The Netherlands contribution, provided by Dutch Space and TNO, was in the areas of Spectral Classification, SAR SPOT autofocus, antenna harness, and ground target co-ordination.

This paper focuses on the main topic of Spectral Classification only. The Spectral Classification-, or SPC mode, is a dedicated mode activated on an operator-selected target, and which is intended to distinguish four target classes: wheeled, tracked (also called 'treaded'), rotary wing, or rotating antenna. This is achieved by producing a Doppler profile, containing modulations due to moving parts of the vehicle, and classifying on the basis of this profile.

The SPC mode is always preceded by FSS (Fast Sector Scan) MTI, which is the mode that detects moving targets and provides the data for target tracking.

\section{Mode DesIGN}

\section{A. Mode description}

The SPC mode is a dedicated mode which is activated by an operator by selecting a target on the operator screen used for SAR and MTI display. Moving targets eligible for classification are detected in the Fast Sector Scan (FSS-MTI) mode and tracked (TRK functionality). This mode activation approach is visualised in Figure 1.

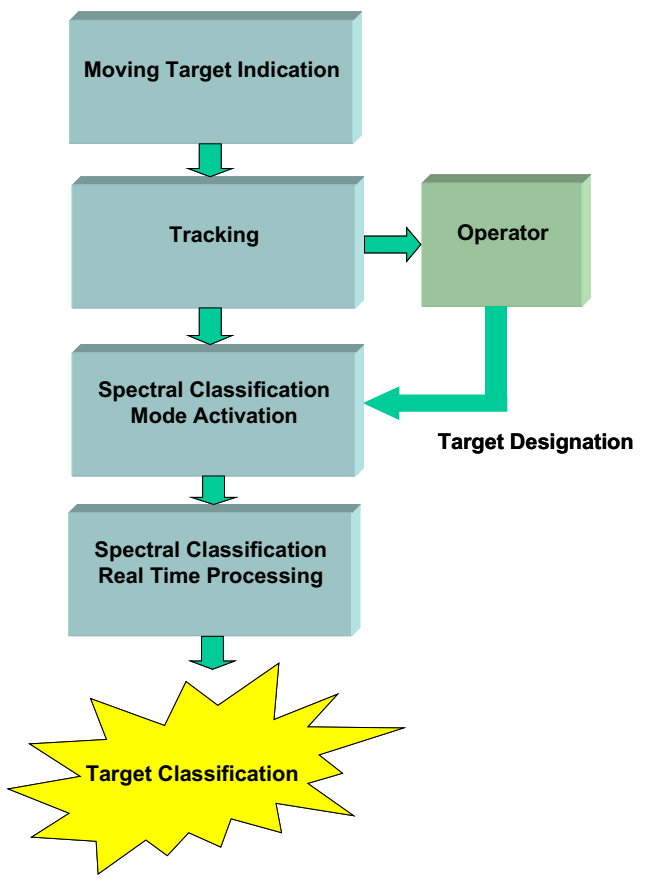

Figure 1 SPC mode activation in flight 
When a target is designated for classification and the mode is activated, an automated sequence is started in which a track prediction is made, and the sensor is switched to SPC mode, illuminating the target at the predicted position with a dedicated waveform. Note that the predicted target position has some uncertainty, and therefore the SPC algorithm must again locate the correct target near the predicted position, after acquiring the data. The target illumination time duration can be selected by the operator in advance, with a minimum of 2 seconds. The acquired data is processed by the real-time processing unit to produce a classification result, which is instantly forwarded to the Tracker and added to the track report. This classification result is then also shown on the operator screen. The result itself consists of target class, i.e. one of the four target classes distinguished and 'unknown', plus a probability vector, providing additional information on, i.e., rough estimation of the relative probability of each class.

\section{B. Mode Design considerations}

Mode Design was mainly driven by two major issues:

- Doppler modulations are relatively weak and unpredictable, typically $20-40 \mathrm{~dB}$ below the main body return signal

- $\quad$ characteristic Doppler width can be quite wide, several $\mathrm{kHz}$ for ground targets, and tens of $\mathrm{kHz}$ for helicopters.

The first issue implies that target-to-noise and target-toclutter ratios must be maximized, leading to coherent integration times which must be much longer than those used for MTI modes; on the other hand, coherent integration cannot be too long because coherence will be lost on a moving target, and especially on the very small Doppler modulation components, which are extremely dependent on target orientation. The selected coherent integration time of 0.4 seconds also necessitates that potential target acceleration is compensated during the integration. The total time-on-target was made operator selectable, in order to be able to adjust it on the basis of flight trial results. In order to achieve maximum target-to-clutter ratio, the three antenna subapertures and receive channels are used to perform clutter rejection by means of Space Time Adaptive Processing (STAP).

The second issue impacts mainly the choice of Pulse Repetition Frequency. Here a trade-off has to be made between the desire for wide unambiguous Doppler bandwidth, and a high probability of helicopter rotor blade flash detection on the one hand, and on the other hand the effects of range ambiguity. Although range ambiguity of targets can be resolved with multiple PRFs, range ambiguity of clutter at very shallow grazing angles (long range) can lead to an unacceptable increase of overall clutter level. A PRF optimisation code was implemented to choose the final PRFs, leading to a division in several range intervals, and a unique set of 5 PRFs, in the range of $2-5 \mathrm{kHz}$, for each of these intervals. During mode activation, the range to the target is known, so the proper set of PRFs is selected, and transmitted in 5 consecutive coherent bursts.
A secondary test mode was implemented as well, in order to enable area illumination without the need for prior target tracking, thus allowing the start of SPC validation as early as possible during the first flight trials. Using GPS data of the targets this mode allowed for on-ground processing, analysis and validation.

\section{CLASSIFICATION APPROACH}

The classification approach was based on experience with ground based spectral classification and makes use of a number of physically motivated spectral features. The most obvious ones are Doppler bandwidth, and modulation frequency. Not only the numerical value of these features, but also the reliability of the estimation is a feature that is used in the classification process. Therefore, reliability measures were also defined and implemented. Based on ground target measurements and simulation, a feature extraction and Bayesian classification scheme was designed and implemented. A practical implementation concern was to allow adjustment of very many classifier parameters, on the basis of flight trial results.

\section{SPC PROCESSING}

\section{A. Processing algorithms}

The processing unit input consists of raw data from a sequence of PRF bursts, including sensor information (waveform parameters, beam position etc) and the predicted track parameters of the designated target. The implemented SPC algorithm chain consists of the following steps:

- calibration, using the results from separate calibration modes

- range compression: for efficiency reasons this is combined with target velocity compensation (using predicted velocity) in the frequency domain, to eliminate range migration

- first stage Doppler processing (short FFT) on subbursts

- target detection, and association with the predicted track

- estimation of actual acceleration

- $\quad$ second stage Doppler processing (long FFT)

- clutter rejection by means of 3-channel Post-Doppler STAP

- classification

Classification consists of:

- acceleration compensation by two methods in parallel 1) spectral scaling, which is most appropriate for ground vehicles, and 2) spectral shifting, which is most suitable for helicopters. Note that at this stage, the target type is still not known.

- feature extraction from the sequence of burst spectra. Spectra are used separately, but also combined to reduce Doppler ambiguity, and burst time sequences are concatenated incoherently to extend the time base for helicopter flash detection: as rotor blade flashes are 
very short, some will be missed in between radar pulses ${ }^{1}$.

- $\quad$ calculation of class probabilities

- choosing the result class

The last step is not just selection of the class with highest probability, because one of the requirements is that the probability of false classification remains very small at all times. This means that in case of insufficient confidence, the result has to be 'unknown'.

The classifier can combine multiple results at the level of features, or at the level of classification results. Either way, classification confidence can be accumulated over the complete target illumination time, or even over multiple classifications on the same track, spread over time.

This processing scheme was designed and implemented off-line by TNO and implemented on the real-time processing unit by Dutch Space. The SPC processing unit was eventually integrated into the SOSTAR-X Radar Processor installed in the SOSTAR-X flight test platform, a Fokker 100 aircraft. This is shown in Figure 2.

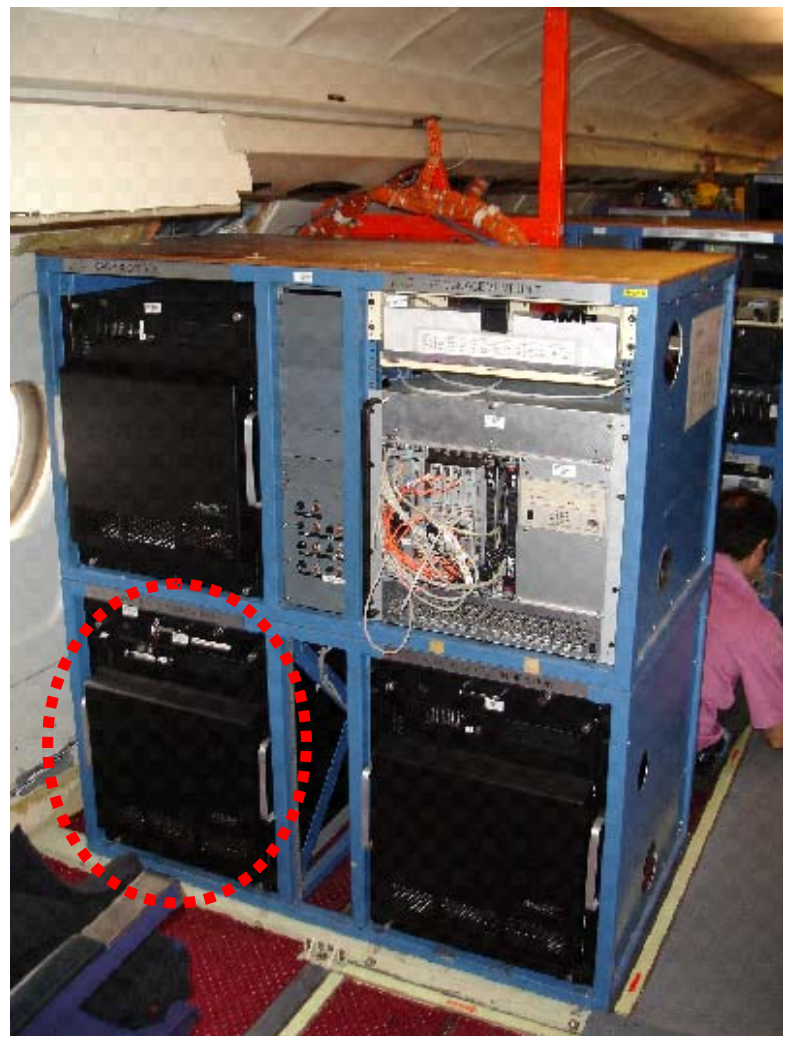

Figure 2 Spectral Classification Real time Radar Processing unit comprising 30 processors (Mercury Computer) installed in the SOSTAR-X flight test platform

\section{B. Real-time SPC algorithm implementation}

The SPC algorithm prototyped by TNO was implemented on the real-time processing unit by Dutch Space. The

\footnotetext{
${ }^{1}$ this can be avoided only with very high PRFs, which are not usable in stand-off geometry, due to associated high range ambiguous clutter
}

functionality of the algorithm steps was assigned to a number of sets of processors. Additionally a number of co-ordinator tasks were identified.

The real-time processing unit consists of a host board and a 30 high performance general purpose PowerPC processors. The host board is connected to the Radar Processor Local Area Network which also includes a Radar Processor Management Unit (RPMU). This RPMU accepts command entered by the Sensor Manager (operator). These commands are related to the state of the radar processor (i.e. whether it should be prepared to accept raw data from mode activations or shutdown) and the actual mode activations (i.e. radar task and notifications on raw data acquisition and radar task completion).

The real-time implementation of the SPC software consists of two programs:

- A program running on the host board accepts, checks and forwards commands from the RPMU to the software running on the PowerPC processors and reports back the state of the real-time processing unit.

- A program running on each of the PowerPC processors providing the following functionality:

- raw data intake and calibration;

- $\quad$ range processing;

- $\quad$ azimuth processing;

- $\quad$ target detection/association;

- $\quad$ STAP processing for targets;

- classification processing on the selected target;

- coordination of PowerPC processor states to be able to report an overall real-time processing unit state;

The actual functionality applicable to a specific PowerPC processor is defined in a configuration file and depends on the number of available PowerPC processors to allow for a scalable implementation.

\section{Flight CAMPAign RESUlts}

Flight campaigns started in the Netherlands, at the Oldebroek Firing Range. Various wheeled, treaded and helicopter targets were deployed during several trials. After the expected initial problem solving, the first clear example of spectral discrimination was obtained in the $4^{\text {th }}$ flight. Figure 3 shows the result on a group of three moving vehicles, one of which is treaded (M109 Howitzer), and two are wheeled. The completely open and uncovered tracks show up very strongly in the range-Doppler image as a range of harmonics. The highest frequency corresponds very well with the velocity of the top part of the tracks, which is twice the speed of the treaded vehicle.

Trials results have also indicated that when tracks are partly covered by metal side plates, spectral features are weaker but often still visible, though less peaked. It is hypothesized that some reflection from tracks may also occur via ground reflection, especially at shallow incidence angles (long range). 

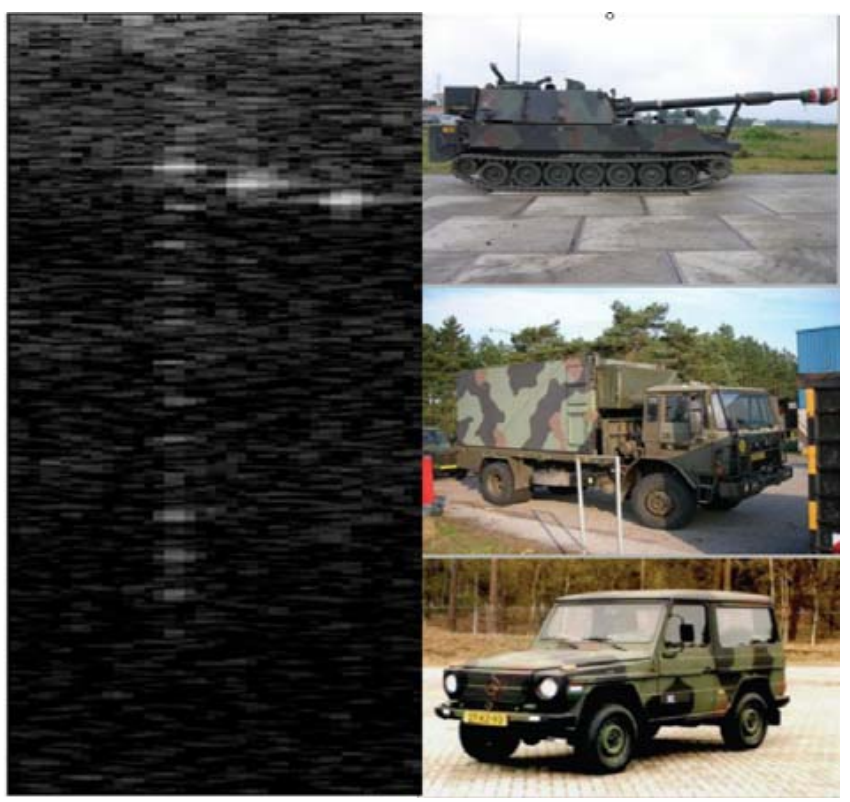

Figure 3 Range-Doppler image (range along horizontal axis, Doppler along vertical axis) with one treaded and two wheeled vehicles as shown

Extensive flight campaigns were done in Germany (Munster, Fassberg) providing valuable data on a wide range of vehicles and several helicopters for further tuning of the classification. The final validation was carried out in France (Angers, Cazaux). Some example results are shown below.

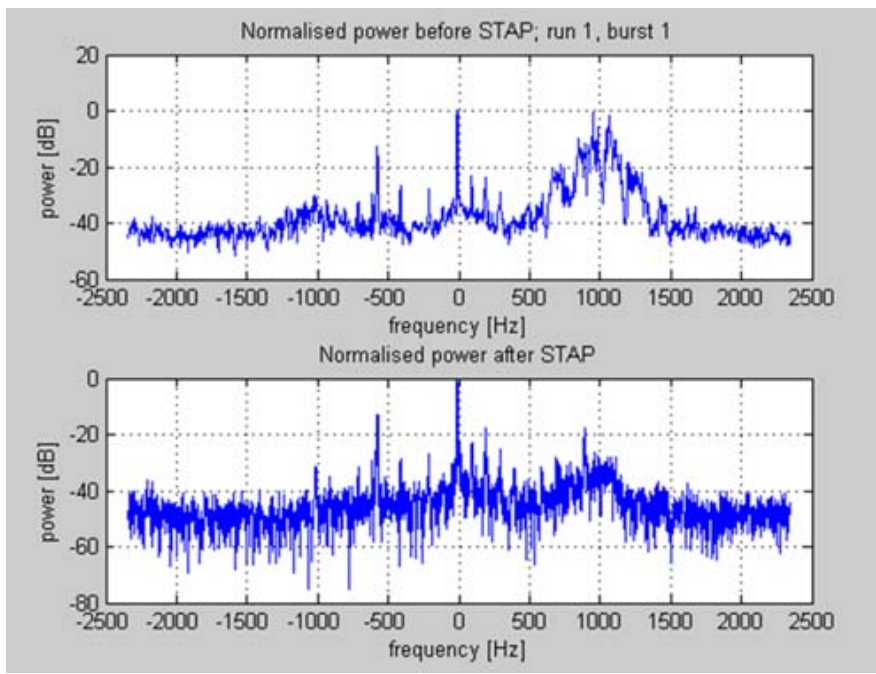

Figure 4 Doppler spectrum of an AMX30 treaded vehicle, before and after STAP processing
Figure 4 shows a Doppler spectrum (from one PRF burst only) of an AMX-30 treaded vehicle, before and after STAP processing. The effect of STAP is apparent in the strong reduction of main lobe clutter (on the right) and side lobe clutter as well. The target spectrum is centred around $0 \mathrm{~Hz}$, because of target motion compensation, while the actual target velocity is about $55 \mathrm{~km} / \mathrm{h}$. Note that the frequency component near $-1000 \mathrm{~Hz}$, which is in this case is quite decisive in the 'treaded' classification, is revealed by the STAP process, as it was before masked by side lobe clutter.

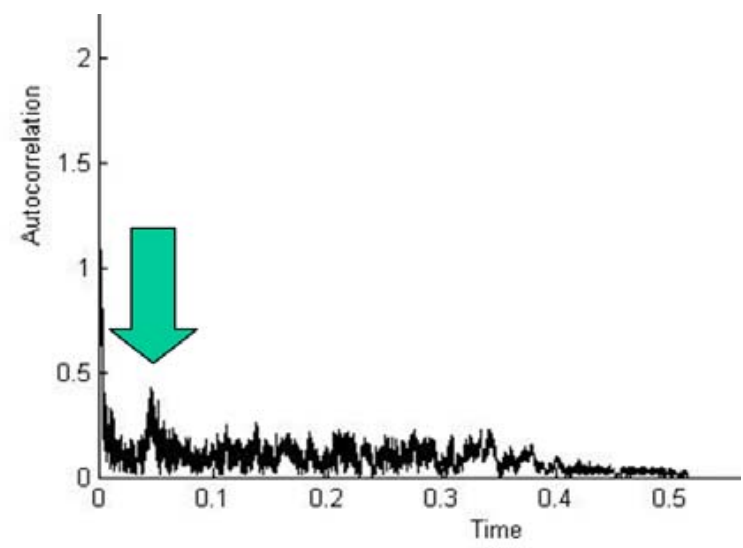

Figure 5 Autocorrelation of a helicopter Doppler spectrum.

Figure 5 shows the autocorrelation of a single helicopter spectrum, revealing a typical modulation peak at about $1 / \mathrm{f}=$ $0.04 \mathrm{sec}$, so $\mathrm{f}=25 \mathrm{~Hz}$. This is a clear indicator of rotor blade modulation.

As expected from physical scattering properties, the detectability of Doppler components from moving parts is quite unpredictable, and combination of several bursts at least has proven to be essential for success; in most tests $2 \times 5$ bursts were used. Overall an acceptable classification score was obtained with this number of bursts, and several ways of further improvement were identified. It was also obvious that a longer time-on-target will significantly increase reliability, due to the unpredictable nature of spectral signatures. For this reason, an alternating SPC/FSS mode was also implemented, allowing a repeated SPC, while FSS detection and target tracking continue.

\section{CONCLUSIONS}

The Spectral Classification mode of SOSTAR-X has demonstrated that successful spectral classification is feasible at long range from an airborne platform, and the general mode design philosophy was validated. Furthermore real-time SPC processing was implemented and demonstrated in-flight for the first time in a European airborne SAR-MTI radar system. 